\title{
L'influence des facteurs macroéconomiques sur les ouvertures d'enquêtes antidumping : Le cas de l'Union européenne et des États-Unis
}

\author{
Mustapha Sadni Jallab ${ }^{1 *}$ \\ Monnet Benoît Patrick Gbakou ** \\ René Sandretto*** \\ * Commission Économique des Nations Unies pour l'Afrique et Centre africain de Politique Commerciale \\ ** Ecole Normale Supérieure Lettres et Sciences Humaines, GATE \\ *** GATE, Université Lyon 2, CNRS, ENS-LSH, Centre Léon Bérard
}

Version révisée-Mai 2008

Résumé :

L’objectif de cet article est d'élargir certains récents travaux qui analysent économétriquement l'influence de quelques variables macroéconomiques sur le nombre des ouvertures d'action antidumping aux États-Unis et au sein de l’Union européenne (Knetter et Prusa, 2003 ; Feinberg, 2005). Les résultats de nos estimations confirment que le taux de change réel exerce une influence similaire pour les deux régions. Les fluctuations du PIB influencent l'ouverture d'enquête antidumping seulement aux États-Unis. A contrario, l'évolution de la production industrielle ne joue pas un rôle significatif pour les États-Unis alors que cet effet est important pour l’Union européenne. Le renforcement de la concurrence internationale apparaît agir de manière significative dans l'accroissement des enquêtes aux États-Unis alors que celui-ci ne semble pas agir de la même manière pour l'Union européenne. Somme toute, une des principales différences entre les États-Unis et l’UE semble être expliquée par les différences de règles et de pratiques mises en place par les autorités de régulations respectives.

Classification JEL : F13 L5- L13

Mots clé : Taux de change Dollar Euro, Enquêtes Antidumping, Modèle Binomial Négatif

\begin{abstract}
:
This paper aims at extending some recent publications about the relationship between antidumping filings and macroeconomic factors by comparing the US and the EU (Knetter et Prusa, 2003; Feinberg, 2005). Results of our estimations confirm that the real exchange rate exerts a similar influence in the two regions. Fluctuations in the real GDP influence antidumping filings only in the US. On the contrary, the evolution of industrial production does not play an important role in the US, while its impact is important in the EU. The reinforcement of international competition appears to significantly increase antidumping filings in the US while this relationship turns out not to be significant in the EU. Finally, some of the most important differences between the US and the EU seem to be explainable by the differences of rules and practices implemented by the regulatory authorities.
\end{abstract}

JEL Classification: F13 L5- L13

Key words: Dollar Euro Exchange Rate, Antidumping initiations, Negative Binomial Model

1 Les opinions émises dans cet article sont strictement celles des auteurs et n'engagent nullement la Commission Economique des Nations Unies pour l'Afrique. Trade, Finance and Economic Development Division, United Nations Economic Commission for Africa, P.O. Box 3001, Addis Ababa, Ethiopia, Phone: 251-115-144-52-12; Fax: 251-115-151-30-38, e-mail: msadni-jallab@uneca.org. Nous remercions des rapporteurs anonymes de la revue pour la richesse et précision de leurs commentaires. 


\section{Ouverture d'enquêtes antidumping et conjoncture macroéconomique : problématique et objectifs}

Un produit doit être considéré comme faisant l'objet d'un dumping s'il est « introduit sur le marché d'un autre pays à un prix inférieur à sa valeur normale », c'est-à-dire « inférieur au prix pratiqué au cours d'opérations commerciales normales pour un produit similaire » ou, à défaut, avec un prix comparable " appliqué aux exportations à destination d'un pays tiers approprié $»^{2}$. Le point de départ de la procédure antidumping consiste en la plainte d'entreprises du pays en nombre suffisant. Aux termes de l'article 6 de l'Accord général, révisé par l'accord sur la mise en œuvre de cet article négocié lors de l’Uruguay round, pour être recevable la plainte doit respecter une série de conditions particulières. En particulier, elle doit bénéficier du soutien d'un nombre minimal d'entreprises de la branche concernée au moins la moitié de celles qui ont exprimé une opinion et représentant au moins $25 \%$ de la production nationale totale de produits similaires. Lorsque la plainte est considérée comme recevable, la suite de la procédure consiste à apprécier la réalité du dumping (sur la base de l'écart entre le prix pratiqué et le prix normal ou " marge de dumping »), à évaluer la réalité du préjudice subi et enfin l'existence d'un rapport de causalité entre le dumping et le préjudice.

En raison des modalités de la procédure elle-même, l'action antidumping est principalement conditionnée par des facteurs d'ordre microéconomique ou sectoriel. En bonne logique, l'étude des actions antidumping devrait donc se situer à ce niveau. Par conséquent, il semblerait que l'analyse des déterminants de ces actions doive être focalisée sur l'évolution la branche et des entreprises concernées. De nombreux travaux ${ }^{3}$ ont été effectués dans cette direction qui soulignent le rôle de multiples variables susceptibles d'influencer la probabilité pour les plaignants d'obtenir gain de cause, ce qui suppose que les autorités nationales reconnaissent effectivement l'existence d'un dommage subi. Cette décision des autorités prend en considération plusieurs critères tels que l'accroissement du taux de pénétration sectoriel des importations, la baisse du niveau de l'emploi et la pression à la baisse des prix dans le secteur concerné, la contraction de leur part du marché domestique, la baisse des profits et du chiffre d'affaires, etc.

Il n'est guère surprenant que les recherches empiriques montrent l'influence significative de ces déterminants microéconomiques et sectoriels. Pourtant, il faut bien admettre que ces résultats sont somme toute assez évidents puisqu'ils sont le reflet de la procédure même et des variables prises en compte par les autorités pour évaluer l'existence d'un dommage. En outré, reconnaître la prééminence des variables microéconomiques et sectorielles ne signifie pas récuser toute influence du contexte macroéconomique. Comparativement aux approches microéconomiques, les essais d'explication des initiations d'action antidumping par des déterminants macroéconomiques sont assez rares. Notre contribution s’inscrit dans la lignée de ces quelques travaux. Considérant comme acquis le rôle des déterminants microéconomiques et sectoriels, nous ambitionnons d'analyser économétriquement l'influence de certaines variables macroéconomiques sur le nombre des ouvertures d'action antidumping aux États-Unis et au sein de l’Union Européenne sur la période récente (1990-

2 Accord sur la Mise en Euvre de l'Article VI de l'Accord Général sur les Tarifs Douaniers et le Commerce de 1994.

3 Parmi la multitude des publications sur ce thème, citons notamment : Hartigan James C., Kamma Sreenivas et Perry P.R. (1989), Feinberg R. et Hirsch B.T. (1989), Lichtenberg F. et Tan H. (1994), Staiger R.W. et Wolak F. A. (1994), Blonigen B.A. et Chad P. Bown (2003), Aggarwal A. (2004) et Feinberg R.M. (2005). 
2002). Plusieurs raisons peuvent justifier a priori l'action de l'environnement macroéconomique sur les ouvertures d'enquêtes :

1. La première explication tient à l'existence de causes macroéconomiques communes à toutes les activités entrant en concurrence avec les produits étrangers et susceptibles d'intensifier la pression concurrentielle avec les producteurs étrangers. On pense bien entendu à l'évolution du taux de change. L'évolution des termes de l'échange pourrait également constituer un bon candidat.

2. Une seconde raison possible tient au fait que l'évolution de l'activité économique dans son ensemble peut renforcer (ou à l'inverse atténuer) l'inclinaison des autorités à donner suite ou non aux demandes d'enquêtes initiées par les producteurs nationaux. Nous pouvons en effet raisonnablement supposer qu'un contexte d'augmentation du chômage et de dégradation de la balance commerciale du pays peut faire évoluer la perception du dommage et de sa gravité par les autorités de réglementation. Par récurrence, il en résulterait une plus forte propension des entreprises à mettre en œuvre la procédure, tant il est vrai qu'il est difficile de dissocier initiation et résolution des actions antidumping. Une plus forte probabilité d'obtenir satisfaction dans un contexte économique donné peut inciter les entreprises à utiliser plus largement la réglementation antidumping.

3. La dernière raison, la plus évidente provient de l'accroissement de la demande de protection avec la détérioration de la situation économique générale. Simultanément, dans une situation de marasme économique et de durcissement de la compétition internationale, les autorités nationales peuvent être tentées d'utiliser la réglementation antidumping non pas au service de la défense de la loyauté, mais à des fins protectionnistes.

Ce risque de détournement de la procédure antidumping est vraisemblablement plus important dans les phases d'évolution défavorable des variables macroéconomiques. En effet, quand la situation économique générale d'un pays se dégrade, la pression exercée par la concurrence extérieure tend à peser plus lourdement qu'en phase de prospérité sur le taux d'utilisation des capacités de production, sur le niveau de l'emploi, sur le taux de profit. Dans de telles circonstances, la probabilité que les autorités de la concurrence concluent à l'existence d'un dommage tend à s'élever. Simultanément, la demande de protection des producteurs domestiques s'accroît elle aussi, sous l'effet conjugué de la détérioration de la situation économique d'ensemble et de meilleures perspectives d'obtenir gain de cause auprès des autorités. En de telles circonstances, ces dernières risquent de prêter une oreille plus attentive et bienveillante à l'argument d'une concurrence étrangère déloyale, déplaçant ainsi la responsabilité des difficultés du moment de l'intérieur (faiblesses de l'appareil industriel, politiques macroéconomiques nuisibles à la compétitivité) vers l'extérieur (déloyauté des concurrents extérieurs). C’est du moins ce que nous voulons tenter de vérifier.

Notre objectif n'est pas de discriminer entre ces trois arguments pouvant justifier l'influence de l'environnement macroéconomique. Il est plus simplement de révéler et évaluer l'action de certaines variables macroéconomiques clés sur les initiations d'actions antidumping, sans contester pour autant que les déterminants de ces actions restent, sans aucun doute, principalement micro et mésoéconomiques. En comparant l'impact de ces facteurs macroéconomiques dans le cas de l'union européenne et des États-Unis, notre but est également de tenter de différentier les pratiques des autorités de régulation de ces deux principales régions du monde. Ce type de préoccupation était déjà en partie présent dans quelques études récentes dans la filiation desquelles s’inscrit notre recherche. 
Après avoir positionné notre contribution par rapport à une revue de la littérature sur le sujet (2), nous présenterons les données, les variables prises en compte et le modèle adopté (3), puis nos principaux résultats (4) et leur interprétation (5) ainsi que nos conclusions (6).

\section{Problématique et revue de la littérature}

Notre étude a pour ambition d'évaluer les relations suivantes :

i) La probabilité d'ouverture de procédures antidumping s'accroît tant aux ÉtatsUnis que dans l’Union européenne, lorsque la valeur réelle de la monnaie du pays considéré s’apprécie.

La littérature sur le « protectionnisme monétaire » montre que le maintien d'une monnaie à un niveau artificiellement sous-évalué peut être un moyen de stimuler les exportations et d'améliorer ainsi de façon déloyale la compétitivité du pays. C'est en gros le grief actuellement fait par les États-Unis à la Chine. Il importe cependant de souligner que le « dumping monétaire » n’est pas visé par les règles antidumping de l'OMC L'évolution du taux de change ne devrait donc pas - en principe, sinon en fait - pouvoir fonder l'application de mesures antidumping, dans la mesure où elle n'est pas le fait des entreprises impliquées et n'est pas non plus du ressort de l'OMC qui n'a pas compétence en matière monétaire internationale. Il n'en reste pas moins que la sous-évaluation d'une monnaie, en réduisant la compétitivité des pays partenaires peut y exacerber la demande de protection. Notre hypothèse est donc que l'appréciation réelle de la monnaie nationale accroît le nombre d'actions antidumping.

ii) La probabilité d'ouverture de procédures antidumping augmente également lorsque la pénétration des importations de produits étrangers devient plus forte c'est-à-dire lorsque la concurrence devient plus intense, même si le degré d'ouverture internationale varie d'un secteur à l'autre. L'explication de cette relation a été donnée précédemment (cf. Section I supra). En effet, nous pouvons raisonnablement supposer que la sensibilité des milieux d'affaires des pays initiateurs aux pratiques de prix déloyales est exacerbée en période de renforcement de la concurrence étrangère et de progression des importations. En outre, dans ce contexte macroéconomique défavorable, les autorités de régulation peuvent faire preuve d'une propension plus élevée à répondre favorablement aux demandes d'ouverture d'enquêtes.

Notre hypothèse est qu'un accroissement du taux de pénétration des importations devrait accroître le nombre des actions antidumping.

iii) Le nombre d'ouvertures de procédures antidumping est une fonction inverse du taux de croissance du PIB. La probabilité d'ouvertures de procédures antidumping augmente avec le ralentissement de croissance économique et, $a$ fortiori, en cas de récession. Parallèlement, le risque de dumping augmente. En effet, lorsque l'économie du pays importateur est déprimée, la demande tend à stagner. Les fournisseurs étrangers seront plus fortement incités à abaisser leurs prix pour tenter de compenser la contraction de leurs débouchés et maintenir le volume de leurs ventes. Enfin, dans une conjoncture économique défavorable, il est plus facile pour le pays importateur d'établir la preuve de l'existence d'un dommage matériel. 
Notre hypothèse est donc qu'un faible niveau d'activité économique (mesuré par l'évolution du PIB) tend à accroître le recours à la procédure antidumping.

A ce jour, trois principales études ont tenté de répondre à ce type de questionnement et notamment aux points 1 et 3 ci-dessus (cf. Tableau 1).. Le travail pionnier de Feinberg (1989) explique à partir d'un modèle Tobit les causes des ouvertures d'enquêtes antidumping aux États-Unis pour la période 1982-1987. Il intègre dans son analyse quatre pays qui sont fréquemment la cible des autorités antidumping américaines : le Japon, le Brésil, le Mexique et la Corée du Sud. L'auteur montre que les fluctuations du taux de change réel constituent un facteur significatif dans les ouvertures d'enquêtes, et tout particulièrement pour le Japon. Plus précisément, il montre que, l'augmentation des procédures antidumping engagées par les États-Unis entre 1982 et 1987 s'explique notamment par la faiblesse du dollar. En outre, il trouve une relation inverse entre le cycle économique et l'ouverture des procédures antidumping américaines ${ }^{4}$. Cependant, la nature discrète des valeurs prises par le nombre d'ouvertures d'enquêtes permet d'émettre des réserves sur la méthode Tobit d'estimation utilisée. Plus récemment, Feinberg (2005) a analysé les enquêtes de dumping trimestrielles ouvertes par les États-Unis sur la période 1981-1998 en utilisant un modèle binomial négatif. Sa conclusion est totalement différente. Il montre qu'une appréciation de $1 \%$ de la valeur réelle du dollar augmente les enquêtes antidumping de 2,06 \%. Les résultats totalement antagoniques sur l'influence du change auxquels parvient R. Feinberg à 16 ans d'intervalle ont de quoi surprendre. Une monnaie faible permet d'exporter moins cher (en devises) et d'importer plus cher (en monnaie nationale), sous condition d'élasticités normales. Cela devrait donc réduire l'incitation à engager des procédures antidumping L'auteur nous livre lui-même une interprétation basée sur l'accroissement du rôle de l'International Trade Commission (ITC) qui a la charge d'évaluer un éventuel préjudice matériel par rapport au Département du Commerce (DOC) chargé de déterminer l'existence - ou non - du dumping entre les deux périodes. Une appréciation du dollar réduit, en effet, la probabilité du dumping si l'on admet que les fournisseurs étrangers répercutent sur les prix l'impact de la dépréciation de leur monnaie (comportement de marge). Dans le même temps, cette hausse du dollar facilite la preuve d'un dommage, dans la mesure où elle renforce l'intensité de la concurrence étrangère. Si cette seconde influence surcompense la première, cela peut signifier que l'influence de l'ITC se renforce comparativement à celle du DOC. L'auteur établit également qu'une augmentation de $1 \%$ du taux de croissance du PIB engendre une baisse de $4 \%$ des enquêtes antidumping.

Knetter et Prusa (2003) se sont aussi intéressés à la relation entre les ouvertures d'enquêtes antidumping et les facteurs macroéconomiques à l'aide d'un modèle binomial négatif pour quatre des principaux initiateurs de mesures antidumping : l'Australie, le Canada, l'Union Européenne et les États-Unis. Dans leur étude, la variable dépendante est le nombre d'enquêtes antidumping ouvertes chaque année au cours de la période 1980-1998. Ils trouvent qu'une appréciation de $1 \%$ du taux de change réel conduit à une augmentation de 2,67 \% des ouvertures d'enquêtes antidumping. Ils montrent également qu'une augmentation de $1 \%$ du taux de croissance du PIB réel moyen engendre une baisse de 7 \% des enquêtes antidumping 5 . Toutefois, de manière surprenante, ces études précédentes ignorent l'influence du taux de pénétration des importations. Or, la prise en compte de cette troisième variable macroéconomique paraît devoir s'imposer pour au moins trois raisons :

- en premier lieu, parce qu'un accroissement du taux de pénétration des importations est

4 Javelot et Siroën (1994) s'étaient déjà intéressés, sans le démontrer, au risque d’un usage abusif des procédures antidumping lorsque l'on prend en compte la volatilité des taux de change.

5 Knetter et Prusa (2002), Table3, troisième colonne. 
l'expression d'un durcissement de la concurrence internationale (ou peut être perçu comme tel).

- $\quad$ en second lieu, parce que la preuve du dommage matériel nécessite de prouver que l'industrie subit une brusque et importante montée des importations.

- $\quad$ en troisième lieu, parce que la nouvelle réglementation antidumping de l’OMC (1994) renforce l'obligation faite au pays plaignant d'établir l'existence d'un lien de causalité entre les importations faisant l'objet du dumping et le préjudice subi par la branche de production concernée.

\section{Déterminants macroéconomiques des ouvertures d'enquêtes antidumping: Une analyse prospective}

Après avoir décrit les données utilisées et les variables retenues dans cette étude, nous explicitons le modèle adopté. Le choix de la méthode d'estimation est justifié logiquement par les résultats d'un test préalable de surdispersion des ouvertures de procédures antidumping. Afin d'établir ces relations, nous commençons par décrire les données utilisées dans cette étude.

\subsection{La description des données}

La majorité des statistiques utilisées dans cette étude provient de la Division de l'Examen des Politiques Commerciales de l'OMC ${ }^{6}$. Les statistiques relatives aux procédures mises en œuvre entre 1990 et 2002 proviennent de la base de données antidumping de l'OMC. La collecte des données est facilitée par l'obligation faite aux pays membres de notifier toute ouverture de procédure antidumping au Secrétariat de l'Organisation ${ }^{7}$. Les figures 1 et 2 présentent la distribution des procédures antidumping durant la période étudiée.

6 La base de données est en partie disponible sur le site de l’OMC. Document référencé sous le numéro suivant WT/TPR/S/102. Nous présentons en annexe les statistiques descriptives de l'échantillon retenu.

7 C'est l'article VI du GATT. 
Tableau 1: Les facteurs influant la décision d’initier une procédure

1. Le taux de pénétration des importations comme variable explicative principale

\begin{tabular}{|c|c|c|c|c|c|c|}
\hline Auteurs & Finger (1981) & $\begin{array}{l}\text { Herander-Schwartz } \\
\text { (1984) }\end{array}$ & Feinberg-Hirsch (1989) & Leipziger-Shin (1991) & Staiger-Wolak (1996) & Krupp (1994) \\
\hline $\begin{array}{l}\text { Facteurs } \\
\text { significatifs }\end{array}$ & 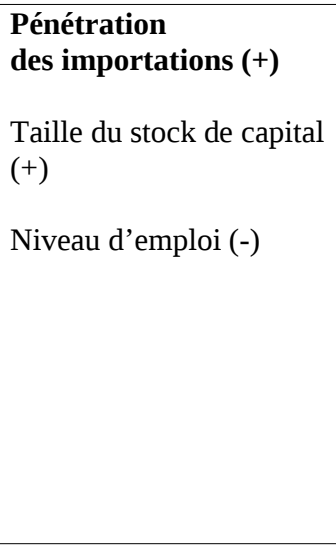 & $\begin{array}{l}\begin{array}{l}\text { Taux de pénétration } \\
\text { des importations } * *(+)\end{array} \\
\text { Retour sur } \\
\text { facteur travail**(+) } \\
\text { Retour sur } \\
\text { facteur capital**(+) } \\
\text { Taux } \\
\text { syndicalisation**(+) } \\
\text { Législation } \\
\text { post-1979 } * *(+)\end{array}$ & $\begin{array}{l}\text { Variation moyenne du taux } \\
\text { de pénétration des } \\
\text { importations sur la période } \\
(+) \\
\text { Intensité en capital (+) } \\
\text { Log nombre d'entreprises } \\
\text { dans la période }(-) \\
\text { Variation moyenne en \% du } \\
\text { nombre d'employés sur la } \\
\text { période (-) } \\
\text { Log nombre d'employé dans } \\
\text { l'industrie }(+) \\
\text { Marge prix-coût (-) } \\
\text { Restrictions sur les quantités } \\
(+)\end{array}$ & 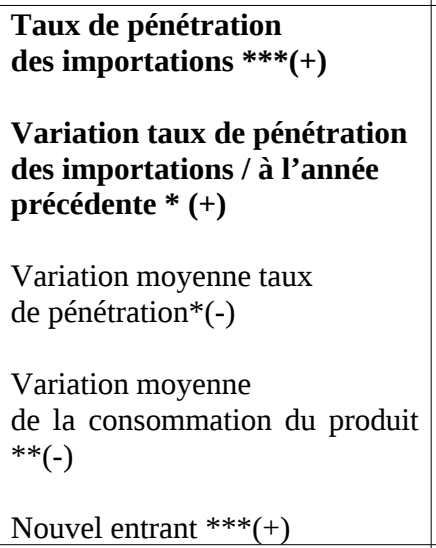 & $\begin{array}{l}\text { Taux de pénétration } \\
\text { des importations (+) } \\
\text { Taux d'utilisation } \\
\text { des capacités (-) } \\
\text { Niveau emploi industrie } \\
(+) \\
\text { Taux de valeur ajoutée (-) }\end{array}$ & $\begin{array}{l}\begin{array}{l}\text { Taux de pénétration } \\
\text { des importations } *(+)\end{array} \\
\text { Niveau d'emploi }(+) \\
\text { Marge dumping>0*(+) } \\
\text { Marge prix-coût **(-) } \\
\text { Législation post- } \\
1979 *(+)\end{array}$ \\
\hline $\begin{array}{l}\text { Facteurs non- } \\
\text { significatifs }\end{array}$ & $\begin{array}{l}\text { Taux de croissance } \\
\text { des importations } \\
(+)\end{array}$ & $\begin{array}{l}\text { Variation taux de } \\
\text { profit (-) }\end{array}$ & $\begin{array}{l}\text { Taux concentration }(+) \\
\text { Taux de syndicalisation }(+) \\
\text { Variation moyenne en \% du } \\
\text { nombre d'employés sur la } \\
\text { période }(+)\end{array}$ & $\begin{array}{l}\text { Variation en \% de la valeur } \\
\text { unitaire des importations (-) } \\
\text { Exportation en provenance d'un } \\
\text { NPI }(+)\end{array}$ & & $\begin{array}{l}\text { Intensité en capital (-) } \\
\text { Salaire moyen }(+)\end{array}$ \\
\hline $\begin{array}{l}\text { Période } \\
\text { étudiée }\end{array}$ & $1975-1979$ & 1976-1981 & $1980-1986$ & 1980-1986 & 1980-1985 & $\begin{array}{l}\text { 1976-1988 } \\
\text { (Industrie chimique) }\end{array}$ \\
\hline
\end{tabular}

Le taux de change comme variable principale

\begin{tabular}{l|l|l|l}
\hline Auteurs & Feinberg (1989) & Knetter et Prusa (2003) & Feinberg (2005) \\
\hline Facteurs significatifs & Taux de change bilatéral ***(+) & Taux de change bilatéral *** $(+)$ & Taux de change bilatéral $* * *(+)$ \\
& Effet temporel du taux de change bilatéral $* * *(-)$ & Taux de croissance du PIB*** $(-)$ & Taux de croissance du PIB $* * *(-)$ \\
\hline Pays étudié & Etats-Unis & Etats-unis & Etats-Unis \\
\hline Période étudiée & $1982-1987$ & $1981-1998$ & $1980-1998$
\end{tabular}

significatif au seuil de $10 \%,{ }^{* *}$ significatif au seuil de $5 \%$, *** significatif au seuil de $1 \%$; entre parenthèse figure le signe de la variable explicative. 
Figure 1: Distribution des procédures initiées par les Etats-Unis pendant la période 1990-2002

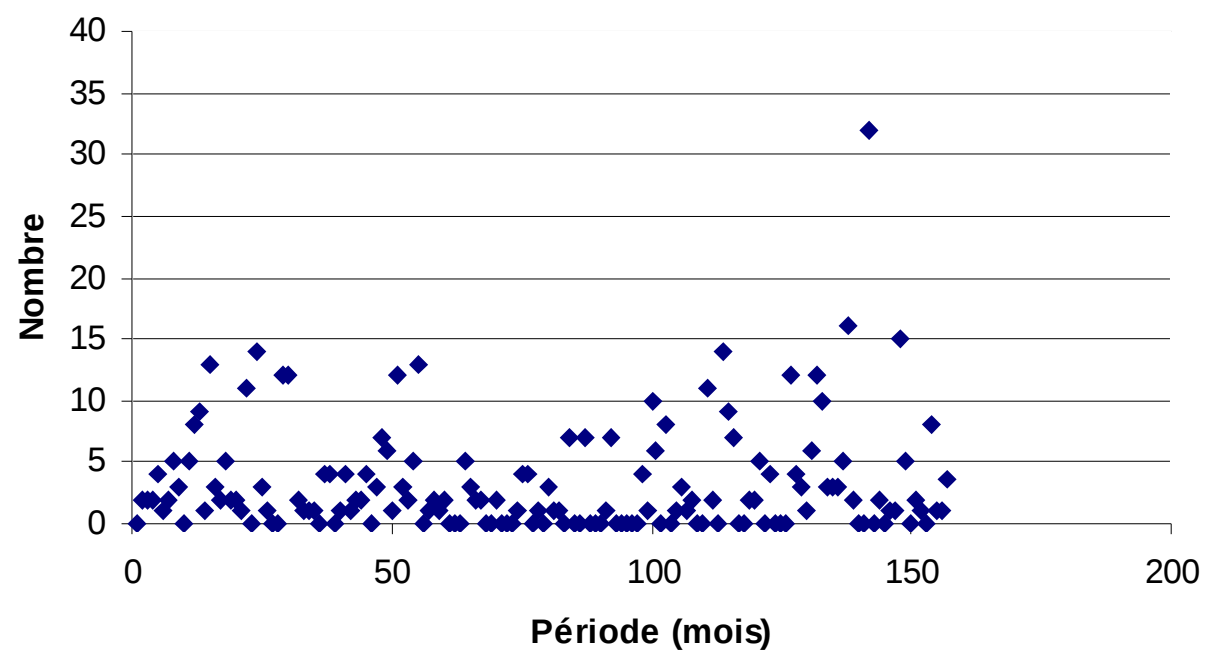

Figure 2: Distribution des procédures initiées par l'Union européenne pendant la période 1990-2002

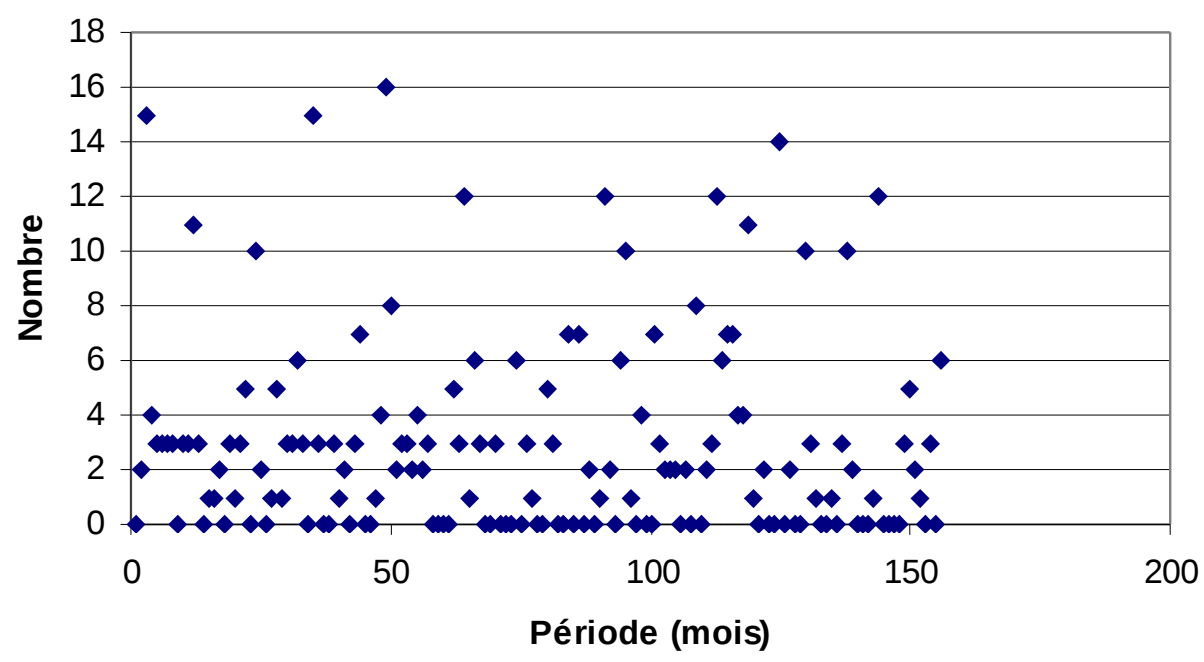

Source: Calculs des auteurs 


\subsection{Les variables}

Toutes les variables utilisées dans l'étude sont évaluées trimestriellement. L’importance du nombre de mois ne comportant aucune ouverture de procédures, nous a conduit à renoncer à une étude mensuelle. Les variables prises en compte dans les modèles utilisés ci-dessous sont calculées à partir de la base de données OLISNET de l'OCDE. Ainsi, nous avons calculé un taux global de pénétration des importations respectivement aux États-Unis et dans l'Union européenne de la manière suivante :

$$
\text { TPIMP }_{i}=\frac{\sum M_{i j}}{D I_{i}}
$$

où $T P I M P_{i}$ est le taux de pénétration des importations du pays $i$ et $M M_{i j}$ est le total des importations de biens et services $j$. Pour l’Union européenne, seules sont prises en compte les importations extra communautaires. L’Union européenne étant considérée comme une seule et même entité commerciale, ce qu'elle est effectivement, à l’instar des États-Unis ${ }^{8}$.

$D I_{i}$ est la demande intérieure du pays $i$.

$D I_{i}=D C P R_{i}+D C P U_{i}+F B C F_{i}$

où :

$D C P R_{i}$ représente les dépenses finales de consommation privées du pays $i$, $D C P U_{i}$, les dépenses finales de consommation publiques du pays $i$ et $F B C F_{i}$, la formation brute de capital fixe du pays $i$.

Les taux de change réels bilatéraux du dollar et de l'euro vis-à-vis des monnaies des cinq pays cibles sont calculés à partir des prix relatifs à la consommation. Les séries de taux de change réel obtenues sont ensuite normalisées en divisant chaque série par sa moyenne. La normalisation vise à éliminer l'effet d'échelle existant entre les divers taux de change.

L’influence du cycle économique est mesurée par les variations du PIB réel ainsi que par celles de l'indice de la production industrielle publié par l'OCDE. De manière plus précise, la variable « cycle économique » est représentée par le taux de croissance moyen du PIB et par l'évolution de l'indice moyen de la production industrielle au cours des trois années antérieures aux ouvertures des procédures antidumping enregistrées chaque trimestre. Le taux moyen de croissance au cours des trois années antérieures est noté TXPIB(-3). L’indice moyen de production industrielle sur la même période est noté INDPROD(-3).La sélection de la période sur laquelle les variables explicatives sont prises en compte est une question importante puisqu'elle conditionne les spécifications apportées aux modèles d'estimation. Tout choix en la matière comporte une part

8 Puisque les actions antidumping initiées dans l’Union européenne ont pour cible des partenaires commerciaux extérieurs à l'Union, il est logique de prendre pour déterminant macroéconomique l'intensification de la pression concurrentielle extra-européenne. 
d'arbitraire. Cependant, retenir un horizon de trois ans est une solution raisonnable dans la mesure où la plupart des pays évaluent les dommages sur une période équivalente. Ce choix est également justifié par le fait que Knetter et Prusa (2003) et Feinberg (2005) ont retenu la même période, ce qui permettra, de comparer nos résultats avec les leurs.

L'analyse économique, mais aussi l'intuition, suggèrent que les périodes de récession renforcent les demandes de protection et favorisent la montée du protectionnisme, alors que les périodes d'expansion sont plus propices à la libéralisation des échanges. Le marasme économique tendrait ainsi, soit à augmenter la fréquence du dumping, soit à multiplier les utilisations des règles antidumping à des fins de protection. Cependant, à l'évidence, les relations de causalité ne sont pas aussi simples dans la mesure où, réciproquement, la politique commerciale influence l'activité économique: la protection renforce la stagnation, voire la récession tandis que l'ouverture commerciale nourrit la croissance. Ainsi, quel que soit le sens de la causalité, doit-on s'attendre à ce que les procédures antidumping fluctuent à l'inverse du cycle des affaires ? Un rapide examen des données semble bien le confirmer. Par exemple, au cours de l'année 1992 - année de conjoncture économique fortement déprimée — on constate que le nombre de procédures antidumping a considérablement augmenté .

De plus, l'accroissement du taux de pénétration des importations tend, lui aussi, à accroître la demande de protection au sein du pays qui le subit. Logiquement, le nombre d'ouvertures de procédures antidumping devrait donc être positivement lié au taux de pénétration des importations du pays considéré. Toutefois, l'évolution des importations d'un pays est liée aux fluctuations de son activité économique. Par conséquent, le taux moyen de pénétration des importations au cours des trois années précédant l'ouverture de l'action antidumping et le cycle économique sont vraisemblablement corrélés. Nous proposerons plusieurs spécifications économétriques afin de neutraliser cette corrélation. Le taux moyen de pénétration des importations antérieur à trois années est utilisé pour les estimations. Il est noté TDPIMP(-3).

Le taux de change réel est un déterminant majeur de la compétitivité. Intuitivement, l'appréciation du taux de change réel tend à détériorer la compétitivité du pays qui le subit et, partant, intensifie les revendications protectionnistes. Nous testons l'existence d'une relation positive entre le taux de change réel et les ouvertures d'enquêtes. Nous retardons la variable taux de change réel d'une année puisque les pays analysent généralement les comportements de prix sur une période d'un an antérieurement à l'ouverture de la procédure antidumping (Knetter et Prusa, 2003). Le taux de change réel retardé d'une année est noté TCR(-1). En outre, pour chacun de ces trois facteurs macroéconomiques, nous tentons de vérifier si l'influence qu'il exerce change au cours du temps et, le cas échéant, dans quelle direction s’opère ce changement. À cet effet, nous introduisons une variable TIME qui correspond à la date de l'ouverture d'une procédure antidumping exprimée en nombre de trimestres écoulés depuis le début de la période d’étude. Le comptage des trimestres est

9 On compte plus de 326 mesures antidumping pour cette année alors que, généralement, ce nombre oscille autour de 200. 
réalisé selon la méthode suivante : nous commençons par attribuer la valeur 1 au premier trimestre de l'échantillon considéré. Le second trimestre de l'échantillon considéré prend la valeur 2, le troisième trimestre la valeur 3 et ainsi de suite. L'interaction entre la variable TIME et les variables explicatives est prise en compte en multipliant chacune de ces variables par le Log de TIME (LogTIME) ${ }^{10}$. Afin de faciliter l'interprétation des coefficients estimés, toutes les variables sont prises en Log, sauf le taux de croissance du PIB. En effet, les séries de taux de croissance du PIB contiennent des valeurs négatives.

\subsection{La procédure d'estimation économétrique}

Le nombre de cas d'ouverture de procédures antidumping est une donnée de type " count data ». C’est une variable de nature discrète. Il est possible de modéliser la survenance d'actions antidumping à l'aide d'un modèle probabiliste basé, soit sur la loi de Poisson, soit sur une loi binomiale négative. La spécification la plus courante des modèles de comptage est le modèle de Poisson. Son extension naturelle, qui permet la prise en compte de l'hétérogénéité non observable, est faite grâce au modèle Binomial négatif. Dans les modèles de régression traditionnels (Modèle Tobit, Cragg...), la distribution de la variable dépendante est dérivée à partir des hypothèses relatives à la distribution du terme d'erreur aléatoire (normalité). Par contre, dans le modèle de Poisson et, d'une manière générale, les modèles de comptage, la distribution de la variable dépendante est donnée directement plutôt que déduite à partir de la distribution du terme d'erreur. Cela implique que le caractère aléatoire du modèle de Poisson est intrinsèque plutôt que résultant d'une erreur stochastique représentant l’hétérogénéité entre les observations. Nous estimons un modèle binomial négatif après avoir effectué des tests qui rejettent le modèle de Poisson sur la base des tests de sur-dispersion de Cameron et Trivedi (1990).

\section{Les résultats économétriques}

\subsection{Les justifications des deux spécifications}

Le comité des pratiques antidumping de l’OMC ne donne pas d'indication précise concernant les périodes à prendre en considération dans le cadre des enquêtes destinées à établir l'existence ou non d'un dumping, pas plus qu'à celles visant à évaluer les dommages. Tout au plus, l'Accord du GATT de 1994 indique-t-il que la durée à prendre en compte dans les enquêtes sur l'existence du

10 La variable TIME est supposée prendre en compte l’idée que, pour les parties plaignantes, l'“apprentissage” administratif des lois commerciales des Etats-Unis a conduit à des changements dans les rôles des déterminants macroéconomiques au cours du temps (Feinberg, 2005). Cet “apprentissage” administratif est fortement lié à l'importance relative des deux agences gouvernementales impliquées dans le processus anti-dumping, l'International Trade Commission du Département du Commerce américain (DOC) et le U.S International Trade Commission (ITC). L'interaction entre les différentes variables explicatives et la variable log TIME nous permet de tester si leurs effets ont changé au cours du temps et dans quelle direction. Le choix de la variable log TIME au lieu de la variable TIME exprimé en niveau vient du fait que les effets d'apprentissage diminuant au cours du temps sont mieux pris en compte dans la spécification log.

La variable TIME est simplement le comptage des trimestres en commençant par le premier trimestre de l'échantillon. 
dumping « devrait normalement être d'un an, mais ne devrait être en aucun cas inférieure à six mois ». Significative de cette absence de lignes directrices bien définies est l'attitude du comité des pratiques antidumping de l'OMC qui estime que les enquêtes visant à apprécier les dommages devraient normalement porter au moins sur trois ans, mais peuvent aussi couvrir une durée inférieure $^{11}$. En définitive, les autorités nationales chargées de l'enquête conservent donc une totale liberté d'adapter ces durées au cas d'espèce considéré. D’où une disparité des cadres réglementaires et des pratiques d'un pays à un autre ${ }^{12}$. Comme nous l'avons souligné précédemment, aux États-Unis, le Department of Commerce (DOC) a la charge d'apprécier l'existence (ou non) du dumping, tandis que l'International Trade Commission (ITC) est responsable de l'évaluation du préjudice subi. Ces deux autorités de réglementation prennent en considération la période des trois années qui précède le dépôt de la plainte.

Au sein de l'Union européenne, la Commission européenne évalue à la fois l'existence du dumping et les préjudices subis par les industries communautaires. La pratique habituelle consiste à sélectionner une période d'enquête (d'une durée minimale de six mois mais qui est de douze mois dans la plupart des cas). Cette première phase permet de calculer la marge de dumping. Dans une deuxième phase, elle prend en considération une période de trois ans pour évaluer le préjudice subi par les industries communautaires suite à l'existence de dumping. Cependant, il se peut que, dans certains cas, l’horizon temporel utilisé pour évaluer le préjudice subi par les industries communautaires soit d'un an seulement ${ }^{13}$. Par conséquent, le fait de considérer une seule année (comme c'est le cas dans certaines affaires examinées par la Commission européenne) ou trois années dans l'évaluation du préjudice n’est évidemment pas indifférent pour l'analyse des déterminants des ouvertures d'enquêtes. C'est la raison pour laquelle nous proposons deux spécifications. La spécification numéro 1 incorpore les variables à l’horizon d'un an alors que la spécification numéro 2 intègre les variables à l’horizon de trois ans. En raison de la corrélation entre le taux de croissance du PIB, le taux de pénétration des importations et l'indice de production industrielle, nous proposons trois modèles binomiaux négatifs dans lesquels ces variables sont présentes séparément. Ces modèles sont appliqués pour chaque spécification aux États-Unis et à l'Union européenne. Soit, au total, six modèles pour chacun des deux pays ${ }^{14}$.

Dans la spécification \#1, nous avons utilisé le taux moyen de croissance du PIB réel, le taux moyen de pénétration des importations et l'indice moyen de production industrielle au cours d'une année antérieure à l'ouverture de l'enquête antidumping considérée (respectivement colonnes i, ii et iii des tableaux 2 et 3). Dans la spécification \#2, nous considérons ces mêmes variables au cours des trois années antérieures à l'ouverture de l'enquête antidumping (colonnes iv, v et vi de ces

11 Recommandation du 5 mai 2000

12 Au niveau des pratiques nationales, la tendance dominante (conforme aux préconisations du comité antidumping de l’OMC) est de retenir une période assez courte pour analyser les pratiques de prix (en général l'année qui précède la plainte) et une période plus longue pour les enquêtes concernant le préjudice (en général 3 ans).

13 Voir les modalités dans la recommandation du 16 mai 2000 de l’OMC G/ADP/6

14 Nous n’avons pas intégré, dans la présentation des résultats, la spécification intégrant le taux de pénétration des importations avec effets temporels pour l’Union européenne. Les résultats n’étaient pas significatifs. 
mêmes tableaux). Le taux de change réel est présent dans toutes les spécifications.

\subsection{Les résultats des estimations du modèle binomial négatif pour les États-Unis}

Afin de faciliter la comparaison de nos résultats avec ceux de Knetter et Prusa (2002) et ceux de Feinberg (2005), nous reportons, dans les tableaux 2 et 3, les ratios des taux d'incidence associés aux coefficients estimés. Selon le modèle de Poisson, le taux d’incidence $\operatorname{Ir}$ (le taux par unité de temps qu'un événement se produise) est une fonction d'un ensemble de variables telle que :

$$
I r=e^{\beta_{0}+\beta_{1} x_{1 j}+\beta_{2} x_{2 j}+\ldots+\beta_{k} x_{k j}}
$$

Le nombre espéré de procédures ouvertes est égal à ce taux d’incidence multiplié par le nombre d'unités de temps au cours de laquelle les observations sont mesurées. Dans notre cas, ce nombre d'unités de temps peu être négligé puisque le nombre de procédures observées est une donnée trimestrielle. Le ratio de taux d'incidence (RTI) est le ratio entre le nombre prédit d'ouvertures de procédures antidumping par le modèle lorsque la variable d'intérêt (par exemple, le taux de change réel, le taux de pénétration des importations...) est d'une unité au-dessus de sa moyenne, toutes les autres variables étant à leurs moyennes, et le nombre prédit d'ouvertures de procédures antidumping par le modèle lorsque toutes les variables sont à leurs moyennes. Par conséquent, si le RTI du taux de change réel est de 1,60, nous dirons qu'un accroissement d'une unité du taux de change réel (ce qui équivaut à une appréciation réelle de $100 \%$ étant donné que nous utilisons des log du taux de change réel) augmenterait le nombre des ouvertures de procédures antidumping de $60 \%$ lorsque toutes les autres variables sont à leurs moyennes. Les $t$-statistiques reportés correspondent à un test d'hypothèse nul RTI $=1$; ce qui impliquerait qu'il n'existe aucune relation entre la variable dépendante et la variable explicative.

\subsection{Les résultats obtenus pour les États-Unis}

\subsubsection{Spécification \#1}

Les variables TXPIB(-1), $\operatorname{LogTPIMP}(-1)$ et $\operatorname{LogINDEXPROD}(-1)$ sont de toute évidence corrélées. Nous avons donc estimé trois modèles où elles sont séparément présentes. Cependant, nous avons pris en compte les interactions de ces variables respectives avec le LogTIME à chaque fois. Le RTI estimé pour le taux de change retardé d'un an LogTCR(-1) est de 1,03 dans les colonnes (i), (ii) et (iii) du tableau 2. C'est-à-dire qu'une appréciation réelle de $100 \%$ du taux de change augmenterait les ouvertures de procédures de $3 \%$. La prise en compte de l'effet temporel montre qu'une appréciation réelle de $100 \%$ du taux de change retardé d’une année réduirait le nombre de procédure de $12 \%$ à $14 \%$ (le RTI est compris entre 0,88 et 0,86 ).

Le taux moyen de croissance du PIB, de même que le taux moyen de pénétration et l'indice moyen de production industrielle ne sont pas significatifs. Leurs effets sont donc considérés 
comme nuls, les RTI sont fixés à 1. En revanche, lorsqu'on prend en compte l'effet temporel, le RTI de LogTIME*TXPIB(-1) est de 0,78. Ce qui implique qu'une réduction de $22 \%$ des ouvertures de procédures est associée à une augmentation de $100 \%$ du taux de croissance du PIB avec effet temporel. L'effet temporel du taux moyen de pénétration des importations est positif et significatif au seuil de $10 \%$. Le RTI de LogTIME*TPIMP(-1) est de 0,31. Une augmentation de 100 \% du taux de pénétration des importations aux États-Unis accroîtrait le nombre d'ouvertures de procédures de 69 \% pour la période considérée.

Somme toute, ces résultats montrent que les variations éphémères du niveau de l'activité économique générale, de l'activité industrielle ou des importations n’ont pas d'impacts sur les ouvertures de procédures antidumping ; Qu'en est-il, en revanche, des effets de ces variables sur des périodes plus longues? Pour répondre à cette question, nous étudions, dans une deuxième spécification, les impacts de toutes ces variables sur une période de trois ans.

\subsubsection{Spécification \#2}

Les colonnes (iv), (v) et (vi) du tableau 2 montrent qu'une appréciation de $100 \%$ du taux de change réel retardé augmenterait les ouvertures de procédures antidumping de $25 \%$. En revanche, une appréciation de $100 \%$ du taux de change réel retardé avec effet temporel LogTIME*LogTCR(-1) réduirait le nombre de procédures de $27 \%$. Les directions d'évolution des variables sont identiques à celles de Feinberg (2005). Cela signifie que la relation positive entre le taux de change réel et l'ouverture d'enquête antidumping est une relation de court terme. Sur une longue période, l'appréciation du taux de change tendrait même à faire baisser le nombre d'ouvertures de procédures. Le RTI du taux moyen de croissance du produit intérieur brut TXPIB(-3) est de 0,09. Une baisse d'une unité du taux de croissance augmenterait donc le nombre d'ouvertures de procédures de $91 \%$ aux États-Unis. Ce résultat est en accord avec nos attentes et avec les résultats des études précédentes : le ralentissement et, a fortiori, la baisse de l'activité économique augmentent les ouvertures d'actions antidumping. Dans les phases d'expansion, on note moins de procédures antidumping. Cependant, lorsque nous prenons en compte l'effet temporel, la relation entre le PIB et le nombre de procédures devient positive : une hausse de $100 \%$ du taux moyen de croissance du PIB augmenterait le nombre d'ouvertures de procédures de $24,6 \%$.

L’impact de l'activité économique est différent lorsqu'on utilise la variable liée à l'indice de production industrielle. En effet, l'impact de LogINDEXPROD(-3) est nul. En revanche, le RTI de LogTIME*LogINDEXPROD(-3) est 1,02. À plus long terme, une augmentation de l'indice de production industrielle de $100 \%$ engendrerait donc un accroissement de $2 \%$ du nombre de procédures antidumping aux États-Unis.

Le taux de pénétration des importations, LogRIMP(-3), a un impact positif et significatif au seuil de $5 \%$. Un taux de pénétration des importations élevé amène donc les États-Unis à ouvrir un plus 
grand nombre de procédures antidumping. Le taux de pénétration des importations a un RTI égal à 1.6015 : une augmentation de $100 \%$ du taux de pénétration des importations entraînerait un accroissement de $60 \%$ du nombre de procédures. Cet effet disparaît à long terme comme le montre le coefficient non significatif de LogTIME*LogTPIMP(-3). Cela signifie qu'à long terme, l'intensité de la concurrence étrangère ne produit pas d'effet significatif sur les initiations d’actions antidumping. L’influence de la compétition est donc essentiellement cyclique.

Tableau 2: Ratio de Taux d’Incidence aux États-Unis

\begin{tabular}{|c|c|c|c|c|c|c|}
\hline \multirow{2}{*}{ Variables } & \multicolumn{3}{|c|}{ Spécification 1} & \multicolumn{3}{|c|}{ Spécification \#2 } \\
\hline & (i) & (ii) & (iii) & (iv) & (v) & (vi) \\
\hline $\operatorname{LogTCR}(-1)$ & $\begin{array}{l}1.0303 \\
(2.749)\end{array}$ & $\begin{array}{l}1.0296 \\
(2.486)\end{array}$ & $\begin{array}{l}1.0302 \\
(2.512)\end{array}$ & $\begin{array}{l}1.2458 \\
(2.921)\end{array}$ & $\begin{array}{l}1.2511 \\
(3.107)\end{array}$ & $\begin{array}{l}1.2544 \\
(3.156)\end{array}$ \\
\hline LogTIME* LogTCR (-1) & $\begin{array}{c}0.8770 \\
(-2.945)\end{array}$ & $\begin{array}{c}0.8763 \\
(-2.737)\end{array}$ & $\begin{array}{c}0.8741 \\
(-2.747)\end{array}$ & $\begin{array}{c}0.7354 \\
(-3.388)\end{array}$ & $\begin{array}{c}0.7308 \\
(-3.566)\end{array}$ & $\begin{array}{c}0.7311 \\
(-3.577)\end{array}$ \\
\hline TXPIB(-1) & $\begin{array}{c}1.00 \\
(0.268)\end{array}$ & & & & & \\
\hline LogTIME* TXPIB(-1) & $\begin{array}{c}0.7815 \\
(-2.417)\end{array}$ & & & & & \\
\hline LogTPIMP (-1) & & $\begin{array}{c}1.00 \\
(0.858)\end{array}$ & & & & \\
\hline LogTIME* LogTPIMP (-1) & & $\begin{array}{l}0.3117 \\
(1.649)\end{array}$ & & & & \\
\hline LogINDEXPROD(-1) & & & $\begin{array}{c}1.00 \\
(1.522)\end{array}$ & & & \\
\hline LogTIME* LogINDEXPROD (-1) & & & $\begin{array}{c}0.9968 \\
(-1.8822)\end{array}$ & & & \\
\hline TXPIB (-3) & & & & $\begin{array}{c}0.0891 \\
(-2.298)\end{array}$ & & \\
\hline LogTIME* TXPIB (-3) & & & & $\begin{array}{l}1.2465 \\
(2.108)\end{array}$ & & \\
\hline LogTPIMP (-3) & & & & & $\begin{array}{l}1.6015 \\
(1.981)\end{array}$ & \\
\hline LogTIME* LogTPIMP (-3) & & & & & $\begin{array}{c}1.00 \\
(1.052)\end{array}$ & \\
\hline LogINDEXPROD(-3) & & & & & & $\begin{array}{c}1.00 \\
(-0.844)\end{array}$ \\
\hline LogTIME* LogINDEXPROD (-3) & & & & & & $\begin{array}{l}1.0179 \\
(1.667)\end{array}$ \\
\hline $\begin{array}{l}\text { Log Maximum de vraisemblance non } \\
\text { contraint }\end{array}$ & -316.324 & -318.566 & -317.981 & -236.217 & -236.63 & -245.799 \\
\hline Test Ratio de Vraisemblance & 96.4656 & 101.4925 & 100.7409 & 18.1267 & 21.0202 & 36.2856 \\
\hline Nombre d'observations & 220 & 220 & 220 & 180 & 180 & 180 \\
\hline
\end{tabular}

Les paramètres estimés sont exprimés sous forme de ratios de taux d'incidence. Les t-statistiques (entre parenthèses) sont exprimés pour un test d'absence d'effet sur les actions antidumping (ce qui correspond à une valeur du RTI de 1.0). 


\subsection{Les résultats obtenus pour l’Union européenne}

\subsubsection{Spécification \#1}

Les colonnes (i), (ii) et (iii) du tableau 3 montrent que le RTI du taux de change réel retardé d'une année est de 1,02. Ainsi, une appréciation de $100 \%$ du taux de change réel augmenterait le nombre des procédures antidumping de $2 \%$. Le RTI de TXPIB(-1) et celui de TPIMP(-1) sont respectivement égaux à 1 . Les variations de court terme de l'activité économique générale et des importations ne semblent donc pas affecter les ouvertures d'actions antidumping dans l'Union européenne. Cette conclusion est nuancée par l'effet de la variable indice moyen de production industrielle. En effet, une augmentation de $100 \%$ de INDEXPROD(-1) diminuerait le nombre de procédures de $14 \%$ (puisque le RTI de l'indice de production industrielle est de 0.856). Au total, seules les variations de court terme de la production spécifique au secteur industriel auront une influence significative au sein de l’Union européenne.

\subsubsection{Spécification \#2}

Le tableau 3 (colonnes iii et iv) montre que le taux de change réel est toujours un déterminant significatif des actions antidumping. Selon notre évaluation, une appréciation de $100 \%$ du taux de change réel conduirait à une augmentation du nombre des procédures antidumping de $13 \%$ à 24 \% (Le RTI varie entre 1,13 et 1,24). Le taux moyen de croissance au cours de trois années qui précèdent l'ouverture d'une procédure a un RTI égal à 1. Les variations cycliques ne semblent donc guère avoir d'influence sur les ouvertures d'enquête dans l'Union européenne. En revanche, le RTI de LogINDEXPROD(-3) est de 0,796. Ainsi, une augmentation de $100 \%$ de l'indice de production industrielle réduirait le nombre de procédures de $20 \%$. Néanmoins, les firmes européennes semblent insensibles au taux de pénétration des importations : nos résultats indiquent que le RTI de cette variable est égal à 1. 
Tableau 3 : Ratio de Taux d'Incidence de l’Union européenne

\begin{tabular}{|c|c|c|c|c|c|c|}
\hline \multirow[t]{2}{*}{ Variables } & \multicolumn{3}{|c|}{ Spécification \#1 : } & \multicolumn{3}{|c|}{ Spécification \#2 } \\
\hline & (i) & (ii) & (iii) & (iv) & (v) & (vi) \\
\hline LogTCR(-1) & $\begin{array}{l}1.0244 \\
(2.472)\end{array}$ & $\begin{array}{c}1.018 \\
(2.570)\end{array}$ & $\begin{array}{c}1.023 \\
(2.516)\end{array}$ & $\begin{array}{l}1.1351 \\
(2.604)\end{array}$ & $\begin{array}{c}1.119 \\
(3.830)\end{array}$ & $\begin{array}{l}1.2431 \\
(3.751)\end{array}$ \\
\hline TXPIB(-1) & $\begin{array}{c}1.00 \\
(0.676)\end{array}$ & & & & & \\
\hline LogTPIMP (-1) & & $\begin{array}{c}1.00 \\
(0.917)\end{array}$ & & & & \\
\hline LogINDEXPROD(-1) & & & $\begin{array}{c}0.856 \\
(-1.965)\end{array}$ & & & \\
\hline TXPIB (-3) & & & & $\begin{array}{c}1.00 \\
(-1.228)\end{array}$ & & \\
\hline LogTPIMP (-3) & & & & & $\begin{array}{c}1.00 \\
(1.217)\end{array}$ & \\
\hline LogINDEXPROD(-3) & & & & & & $\begin{array}{l}0.7962 \\
(-2.37)\end{array}$ \\
\hline $\begin{array}{l}\text { Log Maximum de vraisemblance non } \\
\text { contraint } \\
\text { Test Ratio de Vraisemblance } \\
\text { Nombre d'observations }\end{array}$ & $\begin{array}{c}-208.5098 \\
16.8996 \\
220\end{array}$ & $\begin{array}{c}-209.152 \\
16.335 \\
220\end{array}$ & $\begin{array}{c}-208.83 \\
16.8031 \\
220 \\
\end{array}$ & $\begin{array}{c}-166.22 \\
14.3407 \\
180\end{array}$ & $\begin{array}{c}-163.804 \\
11.794 \\
180\end{array}$ & $\begin{array}{c}-163.61 \\
11.4934 \\
180\end{array}$ \\
\hline
\end{tabular}

\section{Discussion des résultats}

\subsection{Comparaison des résultats entre l’Union européenne et les États-Unis}

Les résultats obtenus permettent de différencier l'influence qu'exercent les facteurs macroéconomiques respectivement aux États-Unis et dans l’Union européenne.

Une première différence tient au fait, que dans l'Union européenne, la prise en compte des effets temporels entraîne la non-significativité des coefficients des variables du modèle. Une explication possible de ce constat est que l'Union européenne a ouvert relativement peu de procédures antidumping sur la période 1990-2002. Le total des ouvertures de procédures n’est que de 133 pour l’Union européenne contre 292 pour les États-Unis.

Le taux de change a un effet significatif aussi bien aux États-Unis que pour l’Union européenne. Les RTI aux États-Unis sont très proches de ceux de l’Union européenne. Pour les États-Unis, 
nous avons établi l'existence d'un effet temporel négatif du taux de change réel retardé d'une année sur les ouvertures de procédures. Cela signifie qu'un maintien du rythme (baisse continue du taux de change) sur toute la période entraîne un impact décroissant sur le nombre des enquêtes. Nous pouvons également constater que la période utilisée pour évaluer le préjudice a une conséquence assez significative sur les ouvertures de procédures d'enquête. Ainsi, pour l'Union européenne, sur une période d’un an (parfois retenue pour évaluer le préjudice), le niveau du RTI du taux de change réel est autour de $2 \%$ alors qu'il est autour de $24 \%$ pour une période de 3 ans retenue pour évaluer le préjudice. Aux États-Unis, lorsque nous retenons une période d'un an pour évaluer le préjudice, nous obtenons un RTI du taux de change réel autour de $3 \%$. Le RTI est autour de $25 \%$ avec une période d'évaluation de préjudice de 3 ans.

Cependant, lorsque la période d'évaluation est de 1 an, le taux de croissance du PIB n'est pas significatif aussi bien pour l'Union européenne que pour les États-Unis. A contrario, lorsque nous retenons une période de 3 ans pour évaluer les préjudices, le taux de croissance du PIB a un effet significatif avec un RTI faible aux États-Unis. Ce qui n’est pas le cas pour l’Union européenne. L'influence des différences de pratiques et des cadres réglementaires en vigueur de part et d'autre de l'Atlantique apparaît donc clairement. En effet, aux États-Unis, contrairement à ce qui se fait dans l'Union européenne, l'ITC utilise systématiquement une période de trois ans pour déterminer le préjudice éventuel. Ces premiers résultats sont confortés par la prise en compte d'un horizon temporel de trois ans. Aux États-Unis, toutes les variables explicatives deviennent alors significatives, tandis que dans l'Union européenne, le PIB n’exerce toujours aucune influence et seule la production industrielle est dotée du signe attendu.

Aux États-Unis, contrairement à l’UE, l'évolution de l'activité économique générale joue un rôle significatif à l'horizon de 3 ans : le nombre des procédures antidumping augmente aux États-Unis avec la baisse du taux de croissance du PIB. Lorsqu'on prend en compte l'effet temporel, on s'aperçoit que le taux de croissance du PIB peut influencer positivement l'ouverture d'enquêtes mais uniquement sur un horizon temporel de court ou moyen terme ${ }^{15}$. Sur une longue période, la relation tendrait à s'inverser. L'intensification de la concurrence internationale constitue également un facteur explicatif des actions antidumping aux États-Unis sur un horizon de trois ans. Comme on pouvait s'y attendre, une augmentation du taux de pénétration des importations aura tendance à accélérer le nombre de procédures et une baisse continue de ce taux sera suivie d'une baisse continue du nombre d'ouverture d'enquête. Enfin, l'évolution de la production industrielle ne permet pas d'expliquer les actions antidumping aux États-Unis. Pour l’UE, au contraire, c’est sans doute le meilleur « candidat » parmi l'ensemble des facteurs explicatifs.

En définitive, trois conclusions principales peuvent être dégagées de cette comparaison :

- Les variations du taux de change sont la meilleure explication commune aux deux zones.

- L'indice de production industrielle est un bon «candidat » pour particulariser l’Union

15 Le coefficient de logTIME*TXPIB(-3) est positif et significatif au seuil de 5\%. 
européenne : il joue dans le sens attendu exclusivement dans l’Union européenne. Au contraire, le taux de croissance du PIB et le taux de pénétration des importations interviennent exclusivement aux États-Unis.

- Le choix de la période d'évaluation du préjudice influence significativement la probabilité d'ouvrir une enquête.

Ainsi, les différences constatées entre les États-Unis et l'Union européenne, quant au jeu des déterminants macroéconomiques, nous semblent pouvoir être expliquées par les différences des règles et des pratiques mises en œuvre par les instances de réglementation pour évaluer le préjudice.

\subsection{Comparaison des résultats obtenus avec ceux de Feinberg $(1987,2005)$ et Knetter-Prusa (2003)}

Le tableau 4 ci-dessous fournit une vision d'ensemble des résultats obtenus comparés aux conclusions des études précédentes. Prusa et Knetter (2003) trouvent qu’une appréciation réelle de $100 \%$ du dollar augmenterait le nombre de procédures de $267 \%$ (avec un ratio de taux d'incidence de 3,67), toutes les autres variables étant à leur moyenne. Feinberg (2005) montre qu'une appréciation réelle de $100 \%$ du dollar augmenterait le nombre de procédures de $129 \%$ (avec un ratio de taux d'incidence de 2,29). Nos estimations vont dans le sens des études précédentes, mais avec des ratios de taux d'incidence plus faibles. Plusieurs justifications peuvent être mises en avant :

- Notre période d'étude utilise des données trimestrielles et s'étend sur une période plus courte (1990-2002), alors que les deux études citées s’intéressent à la période 1980-1998. Sur une période plus longue 1981-1998, le nombre de procédures observées est plus important.

- De plus, leurs régressions utilisent des modèles binomiaux négatifs à effets aléatoires sur données de panel. Nos tentatives pour effectuer de telles régressions se sont avérées infructueuses sur la période 1990-2002. Les données que nous avons récoltées sont inconsistantes avec un modèle binomial à effets aléatoires sur une période d'étude assez courte. 
Tableau 4 : Sens des relations mises en évidence dans l’étude économétrique

\begin{tabular}{|c|c|c|c|c|c|}
\hline Variables macroéconomiques & \multicolumn{3}{|c|}{ États-Unis } & \multicolumn{2}{|c|}{ Union européenne } \\
\hline & Spécification \#1 & Spécification \#2 & F-PK ${ }^{16}$ & Spécification \#1 & Spécification \#2 \\
\hline $\begin{array}{l}\text { Taux de change sans effet } \\
\text { temporel }\end{array}$ & $+* * *$ & $+* * *$ & $+* * *$ & $+* *$ & $+* * *$ \\
\hline $\begin{array}{l}\text { Taux de change avec effet } \\
\text { temporel }\end{array}$ & $-* * *$ & -*** & $-* * *$ & -** & $-* * *$ \\
\hline PIB sans effet temporel & + & $-* *$ & $-* *$ & + & - \\
\hline PIB avec effet temporel & $-* *$ & $+* *$ & $+* *$ & - & - \\
\hline $\begin{array}{l}\text { Production industrielle sans } \\
\text { effet temporel }\end{array}$ & + & $+* *$ & nd & _** & -** \\
\hline $\begin{array}{l}\text { Production industrielle avec } \\
\text { effet temporel }\end{array}$ & $-*$ & - & nd & - & - \\
\hline $\begin{array}{l}\text { Taux de pénétration des } \\
\text { importations sans effet } \\
\text { temporel }\end{array}$ & + & $+* *$ & nd & -* & $-* *$ \\
\hline $\begin{array}{l}\text { Taux de pénétration des } \\
\text { importations avec effet } \\
\text { temporel }\end{array}$ & $+*$ & + & nd & ns & ns \\
\hline
\end{tabular}

$* * *=$ significatif au seuil de $1 \%$. **= significatif au seuil de $5 \%$. * significatif au seuil de $10 \%$.

nd signifie que ces variables n’ont pas été étudiés dans les travaux antérieurs. ns signifie non significatif.

\subsection{Conclusion de l'analyse des résultats économétriques}

Nous avons montré que les variables macroéconomiques ont des effets différents sur les ouvertures de procédures antidumping aux États-Unis et dans l'UE. Tout d'abord, une appréciation du taux de change réel a un impact positif sur les ouvertures de procédures aux ÉtatsUnis et au sein de l'Union européenne. Cependant, l'ampleur de l'effet est beaucoup plus importante aux États-Unis et l'effet temporel du taux de change est présent uniquement aux ÉtatsUnis. Ensuite, le cycle économique (mesuré par le taux de croissance du PIB) a un impact sur les ouvertures de procédures uniquement aux États-Unis. L'intensification de la concurrence étrangère (mesurée par le taux de pénétration des importations) accroît aussi les ouvertures de procédures aux États-Unis. Ces dernières sont aussi influencées par l'évolution à très court terme et/ou à court terme de l'activité économique générale américaine, tandis qu'elles dépendent plus spécifiquement de la conjoncture propre au secteur industriel dans l'Union européenne. Ces distinctions montrent l'influence des différences institutionnelles, notamment au niveau des règles qui prévalent au niveau de la procédure antidumping de part et d'autre de l'Atlantique. La différence concernant les périodes de référence dans l'appréciation des dommages est perceptible à travers nos résultats (période plus courte dans le cas de l’Union Européenne de l'ordre de 15 mois contre 3 ans aux États-Unis). Il semble aussi que la procédure européenne soit moins efficace - ou dotée d'un moindre « effet de protection » — que la procédure américaine lorsque

16 Cette colonne permet de comparer nos résultats avec ceux obtenus par Feinberg $(1989,2003)$ et ceux de Prusa et Knetter (2003). 
l'on considère l'impact du cycle économique, l'influence du change et, plus encore, celle de la pression concurrentielle. Pourtant, paradoxalement, la procédure européenne semble plus sélective, plus ciblée que la procédure américaine (pas de relation significative avec le PIB dans l'UE, mais relation avec la production industrielle alors que c'est l'inverse aux États-Unis ${ }^{17}$. Audelà de l'explication des ouvertures de procédures antidumping, un élargissement de l'étude est nécessaire pour prendre en compte les conditions de la concurrence mondiale. En effet, la définition du dumping telle qu'elle est inscrite dans les Accords de l'OMC ne mentionne pas que l'appréciation du taux de change peut justifier d'emblée l'ouverture d'une enquête antidumping.

\section{Conclusion}

De manière différente aux États-Unis et dans l’Union européenne, notre étude montre que les variables macroéconomiques influencent significativement les ouvertures de procédures antidumping. C'est le cas, en particulier du taux de change réel (dans les deux régions), du PIB et du taux de pénétration des importations (aux États-Unis), ou de l'évolution d'ensemble de la production industrielle (dans l'UE). En outre, les effets produits ne sont pas les mêmes à court terme et à long terme. L'interprétation de ces résultats n'est pas évidente. Elle apporte quelques zones de clarté, mais elle laisse aussi des zones de pénombre.

1. La conclusion qui ressort avec le plus de netteté est que les discordances observées entre les deux régions du monde sont largement explicables par les différences dans les réglementations, les procédures et les pratiques nationales en matière d'action antidumping. C'est sans doute là l'explication la plus crédible des différences d'effets observés aux États-Unis et dans l’UE, lorsque l'on fait varier la période de référence pour l'appréciation des dommages.

2. Le "clair-obscur » qui subsiste au terme de notre étude concerne l'explication même de l'impact des variables macroéconomiques. Ainsi que nous l'avons souligné, cette influence peut résulter :

- D’une part, de l'existence de facteurs macroéconomiques communs, pouvant agir sur l'ensemble des secteurs d'activité. C'est le cas par exemple du taux de change. Il n'est donc guère surprenant que le taux de change soit la variable dont les impacts sont les plus similaires de part et d'autre de l'Atlantique.

- D’autre part, d'une sensibilité particulière des autorités au contexte macroéconomique. Celles-là témoigneraient en quelque sorte d'une propension différente à accepter et donner suite aux plaintes selon que le contexte macroéconomique est favorable ou défavorable. En somme, les autorités auraient un comportement différencié selon la conjoncture globale dans leur évaluation de l'existence et du dumping et de son caractère dommageable, soit à leur insu - en toute bonne foi, soit consciemment, avec une visée délibérément protectionniste.

17 Bien entendu, pour pouvoir être pleinement validée, cette interprétation nécessiterait d'être consolidée par une analyse plus approfondie. 
- Enfin, le choix de l'échantillon (1990-2002) apporte un éclaircissement pour cette période, mais qui ne nous permet pas de généraliser nos résultats.

Pour mettre en évidence le poids respectif de ces motifs, une analyse plus fine au niveau sectoriel et temporel, voire même au cas par cas au niveau des entreprises, serait nécessaire, croisant une pluralité de critères, tels que l'importance de la marge de dumping retenue (et son évolution avec la conjoncture), la contribution du secteur concerné au PIB et à l'emploi national, son niveau de concentration industrielle, le degré de libéralisation des échanges, le taux de syndicalisation, etc. Une telle investigation sort du cadre de l'approche purement macroéconomique adopté dans notre étude. Cependant, elle en constituerait un prolongement à la fois naturel et indispensable. Bien que notre analyse ne permette pas de l'établir explicitement, l'hypothèse du détournement de la procédure à des fins de protection par les autorités apparaît plausible, en particulier lorsque l'on constate que des marges de dumping minimes de 2 à $3 \%$ - inférieur au degré de précision des données — suffisent à déclencher l'application de droits antidumping.

Le risque que la réglementation antidumping soit détournée de sa finalité pour en faire un instrument de " protectionnisme furtif ${ }^{18}$ est aujourd'hui largement dénoncé, dans un contexte de durcissement de la compétition internationale. La procédure telle qu'elle a été élaborée et mise en application facilite incontestablement le développement d'un protectionnisme déguisé derrière l'uniforme de la préservation de la loyauté et du maintien de l'ordre commercial international. Ce risque de « dévoiement » s'explique en grande partie par le fait que si les règles antidumping de l'OMC sont internationales, leur application est purement nationale. Il appartient donc aux autorités du pays «victime » de se prononcer sur l'existence et le caractère préjudiciable du dumping et de décider des mesures à prendre. Les investigations étant totalement à la discrétion des autorités, les entreprises à l'origine des plaintes ont une forte probabilité de pouvoir bénéficier d'une protection et même si ce n'est pas le cas, les études empiriques existantes montrent que le seul fait d'engager la procédure procure un effet de protection en provoquant une baisse des importations ${ }^{19}$. En outre, les droits antidumping, contrairement aux droits compensateurs (antisubvention) sont ciblés sur des firmes et non des gouvernements. Par suite, ils ne sont soumis ni à la règle de la nation la plus favorisée ni au principe de réciprocité (contrairement à la clause de sauvegarde), ce qui les rend particulièrement attractifs comme instruments de protection :

- en raison de leur remarquable sélectivité (non seulement au niveau des pays, mais aussi à celui des secteurs et des firmes) ;

- parce qu'ils n'exposent pas le pays qui les applique à l'obligation de négocier des compensations. Ces caractéristiques confèrent aux actions antidumping les propriétés d'un instrument de protection opaque (protection non transparente et non visible en tant que telle) et non soumise aux contraintes que les règles de l'OMC (non-discrimination et réciprocité) imposent aux autres instruments. Seule subsiste la possibilité d'un recours des pays affectés pour procédure abusive, arbitré par l'OMC.

18 Cf. Sandretto (1998)

19 Prusa T. (1999) 
L'aspect le plus étonnant est que cette protection particulière puisse être mise en application en exploitant des règles destinées à construire un système commercial multilatéral ouvert. Une révision des règles antidumping de l'OMC s'impose afin d'en améliorer l'efficacité et éviter les dérives auquel elles peuvent donner lieu. On peut penser par exemple à l'adoption de mécanismes plus rapides de règlement des différends pour lutter contre les investigations injustifiées (raccourcissement des délais de mise en place du panel, création d'un corps d'experts permanents, etc.) ou encore à l'institution de dédommagements financiers suffisamment dissuasifs au bénéfice des entreprises injustement et, a fortiori, abusivement poursuivies. Toutefois, une réforme en profondeur passe par un changement radical des règles internationales qui mette fin à la confusion des rôles de juge et partie en transférant la mise en œuvre de la procédure du niveau national au niveau multilatéral. Compte tenu des oppositions unanimes qu'elle soulève, une telle réforme reste une perspective très aléatoire, ou du moins de long terme, peut-être même de très long terme. 


\section{Bibliographie}

Aggarwal A. [2004], Macro Economic Determinants of Antidumping: A Comparative Analysis of Developed and Developing Countries, World Development, vol. 32, issue 6, 1043-1057.

Blonigen B.A. et Chad B. [2003], Antidumping and Retaliation Threats, Journal of International Economics, vol 60, n², 249-273.

Blonigen B.A., Gallaway M.P. et Flynn J.E. [1999], Welfare Costs of the U.S. Antidumping and Countervailing Duty Laws, Journal of International Economics, vol 49, n², 211-244.

Blonigen B.A. et Haynes S.E [2002], Antidumping Investigations and the Pass-Through of Exchange rates and Antidumping Duties, American Economic Review, vol 92, n 4, 1044-1061.

Cameron C.A. et Trivedi P.K. [1986], Econometric Models Based on Count Data: Comparisons and Applications of some Estimators and Tests, Journal of Applied Econometrics, vol 1, $\mathrm{n}^{\circ} 1$, 29-53.

Cameron C.A.et Trivedi P.K. [1990], Regression Based Tests for Overdispersion in the Model Poisson, Journal of Econometrics, vol 46, n³, 347-364.

Feinberg R.M. [1989], Exchange Rate and Unfair Trade, Review of Economics and Statistics, vol71, n4, 704-707.

Feinberg R.M. [2005], U.S. Antidumping Enforcement and Macroeconomic Indicators Revisited: Do Petitioners Learn?, Review of World Economics, vol. 141, n4, 612-622.

Feinberg R.M. [2006], Exploring the Patterns and Determinants of U.S. Antidumping Actions against Latin American Imports, 1980-2004. CEPAL - Serie Comercio internacional. United Nations Publication, $n^{\circ} 78$, Santiago, September, 1-30.

Feinberg R.M. et Hirsch B. [1989], Industry Rent-Seeking and the Filing of 'Unfair' Trade Complaints, International Journal of Industrial Organization, vol 7, n³, 325-340.

Feinberg R.M et Reynolds K. [2006], Friendly Fire? The Impact of US Antidumping Enforcement on US Exporters. American University, Working paper n²006-04, April.

Finger J.-M. [1981], The Industry-Country Incidence of Less-than-Fair-Value Cases in US Import Trade, Quarterly Review of Economics and Business, vol 21, n5, 260-279.

Finger J.M., Ng F. et Wangchuk S. [2001], Anti-Dumping as Safeguard Policy, Working paper n ○ 2730, Washington DC, World Bank.

Hartigan J.C., Kamma S. et Pery P.R. [1989], The Injury Determination Category and the Value of Relief from Dumping, Review of Economics and Statistics, vol 71, 183-186.

Hausman J., Hall B. et Griliches Z. [1984], Economic Models for Count Data with an Application to the Patents-R\&D?, Econometrica, vol 52, n²4, 909-938. 
Herander M. et Schwartz, J.-B. [1984], An Empirical Test of the Impact of the Threat of US Trade Policy: The Case of Antidumping Duties, Southern Economic Journal, vol 51, n¹, 59-79.

Javelot S. et Siroën J.-M. [1994], Les nouveaux instruments de politiques commerciales, Revue Economique, Mai, $\mathrm{n}^{\circ} 45$.

Knetter M. et Prusa T.J. [2003], Macroeconomic Factors and Anti-Dumping Filings: Evidence from Four Countries, Journal of International Economics, vol 61, n²1, 1-17.

Krupp C. [1994], Antidumping Cases in the US Chemical Industry: A Panel Data Approach, Journal of Industrial Economics, vol 42, n²1, 299-311.

Leidy M. [1997], Macroeconomic Conditions and Pressures for Protection Under Antidumping and Countervailing Duty Laws: Empirical Evidence from the United States, IMF Working Paper 44, n²1, March, 132-145.

Leipziger D.M. et Shin H.J. [1991], The Demand for Protection: A Look at Antidumping Cases. Open Economies Review, vol 2, 27-38.

Lichtenberg F. et Tan H. [1994], An Industry-Level Analysis of Import Relief Petitions Filed by US Manufacturers, 1958-1985. in H. Tan and H. Shimada [ed], Troubled Industries in the UnitedStates and Japan, New-York St Martin Press, 161-188.

Prusa T.J. [1992], Why Are So Many Antidumping Petitions Withdrawn?, Journal of International Economics, vol 33, n¹-2, 1-20.

Prusa T.J. [1994], Pricing Behavior in the Presence of Antidumping Law, Journal of Economic Integration, vol 9, $\mathrm{n}^{\circ} 2,260-289$.

Prusa T.J. [1999], On the Spread and Impact of Antidumping. NBER Working Paper n W7404.

Sandretto R. [1998], Le protectionnisme au tournant du siècle : Opacité et furtivité, Revue Études Internationales, Institut québécois des hautes études Internationales, Vol XXIX, n²2, Juin.

Siroën J.-M. [1998], Monopoles naturels, ouverture commerciale et gains de l'échange, Economie Internationale, $\mathrm{n}^{\circ} 75$, 3ème trimestre.

Staiger R.W. et Wolak F.A. [1994], Measuring Industry-Specific Protection: Antidumping in the United States, Brookings Papers on Economic Activity, Vol (Microeconomics), 51-103.

Staiger R.W et Wolak F.A [1996], Differences in Uses and Effects of Antidumping Law Across Import Sources, in A. Krueger [ed], The Political Economy of American Trade Policy, Chicago, University of Chicago Press/NBER, 385-415. 


\section{Annexe :}

Tableau A : Statistiques descriptives des variables utilisées dans l'étude

\begin{tabular}{|l|l|c|c|c|c|}
\hline Pays & & Total PAD & TPIMP & TDCR & TXPIB \\
\hline Union européenne & & & & & \\
& & & & & \\
& Moyenne & 2,89 & 0,296 & 0,8988 & 1,222 \\
\hline & Ecart-Type & 3,608 & 0,0338 & 0,1257 & 0,595 \\
\hline & Min & 0 & 0,25177 & 0,7135 & 0,103 \\
\hline & Max & 16 & 0,3714 & 1,1721 & 3,284 \\
\hline \multirow{2}{*}{ États-Unis } & & & & & \\
& & & & & \\
& Moyenne & 3,914 & 0,121 & 1,14059813 & 1,236 \\
\hline & Ecart-Type & 6,956 & 0,0154 & 0,14690605 & 0,492 \\
\hline & Min & 0 & 0,0126 & 0,85316952 & 0,041 \\
\hline & Max & 49 & 0,148 & 1,4015417 & 2,145 \\
\hline
\end{tabular}

